\title{
A Rice Black-Streaked Dwarf Virus Replication Curve Model to Evaluate Maize Rough Dwarf Disease Resistance
}

\author{
Han Xiaohua, ${ }^{1}$ Chen Tingmu, ${ }^{2}$ Yue Runqing, ${ }^{1}$ Guo Shulei, ${ }^{1}$ Xu Mengmeng, ${ }^{1}$ Lu Caixia, ${ }^{1}$ Fan Yanping, ${ }^{1}$ Chen Nana, ${ }^{1}$ Liu Lu, \\ Fu Xiaolei, ${ }^{1}$ Chi Haifeng, ${ }^{1}$ Guo Xinhai, ${ }^{1}$ Xia Yan, ${ }^{1}$ and Tie Shuanggui ${ }^{1, \dagger}$ \\ ${ }^{1}$ The Cereal Crops Institute, Henan Academy of Agricultural Sciences/Henan Key Laboratory of Maize Biology, Zhengzhou, \\ Henan Province 450002, China; and ${ }^{2}$ Lianyungang Academy of Agricultural Sciences, Lianyungang, Jiangsu Province \\ 222000, China
}

\begin{abstract}
Resistance to maize rough dwarf disease (MRDD), a major cause of crop losses, depends on external conditions such as the virus transmission period and the rate of viruliferous small brown planthoppers, Laodelphax striatellus. The precise identification of MRDD contributes to the utilization of resistant germplasm and the cloning of resistant genes. In this study, eight maize varieties were artificially inoculated in a greenhouse with viruliferous planthoppers. The viral titers in maize seedlings were detected by quantitative fluorescence RT-PCR, and the viral replication curves were analyzed by regression. A logistic model fit the Rice black-streaked dwarf virus (RBSDV) replication data for five susceptible varieties well, whereas a

linear model fit the data for three resistant varieties. Among the five susceptible varieties, the time points with the maximum replication rates $\left(t_{I P}\right)$ of the highly susceptible Ye478 and XH6 were significantly earlier than those of the three susceptible varieties, Mo17, Zheng58, and Zhengdan958. P138, the most highly resistant variety, had the lowest slope of the best fit line, followed by moderately resistant Chang7-2 and Dan 340. The RBSDV replication curve model developed in this study can accurately identify the resistance of maize germplasm to MRDD at the molecular level. Our results also suggested that $t_{I P}$ and the slope of the RBSDV replication curve can be considered new criteria to evaluate maize resistance to MRDD.
\end{abstract}

Maize rough dwarf disease (MRDD) is one of the most serious viral diseases affecting maize worldwide, resulting in huge crop losses. Maize rough dwarf virus (MRDV), Mal de Rio Cuarto virus (MRCV), and Rice black-streaked dwarf virus (RBSDV) are three pathogens that cause MRDD (Fang et al. 2001; Lenardon et al.1998; Louie et al. 1999). Previous sequence analysis of dwarfing disease pathogens in maize in China have indicated that RBSDV is a causal agent and its vector is the small brown planthopper (SBPH) (Chen et al. 2004; Fang et al. 2001; Zhang et al. 2001; Zhou et al. 1996). The cultivation of resistant varieties is the foundation for disease management (Zhou et al. 1996).

The identification of resistant germplasm, the basis for breeding varieties with disease resistance, includes two major components, i.e., the inoculation method and criteria for evaluating resistance. Two inoculation methods are widely used: natural field assessments, which are susceptible to natural variation, including variation in the transmission period, population density of SBPH, and the rate of viruliferous SBPH, etc.; and artificial inoculation of viruliferous planthoppers, which is considered more accurate. Disease incidence rate and disease severity index (DSI) based on biological symptoms in plants are common criteria used to evaluate resistance, but results based on these properties are not replicable owing to their environmental sensitivity. For example, in field studies, DSI or disease incidence rate was used to evaluate the severity of MRDD. Lu et al. (2001) identified P138 as resistant and Dan340 and Mo17 as highly susceptible. Chen et al.(2006) reported that P138, Dan340, Mo17, and Zheng58 are highly resistant, resistant, susceptible, and susceptible, respectively. Moreover, Xie et al. (2009) characterized Zheng58 as highly susceptible. P138 has been identified as highly

${ }^{\dagger}$ Corresponding author: S. G. Tian; tiesg05@163.com

Funding: This work was supported by the Henan Fundamental and Frontier Research Fund, no. 162300410178, and the Henan Science and Technology Cooperation Project, no. 182106000040.

The authors declare that they have no competing interests.

Accepted for publication 18 August 2018.

() 2019 The American Phytopathological Society resistant, and Zheng58 has been identified as susceptible by artificial inoculation in a greenhouse (Chen et al. 2007). In another study, P138 was identified as resistant in a virus-prone field (Xue et al. 2011).

The precise identification of MRDD affects not only the screening and utilization of resistant germplasm but also the precision of gene mapping. No rough dwarf disease resistance genes have been cloned (Di Renzo et al. 2004; Luan et al. 2012; Shi et al. 2012) owing to the limitations of current methods for detecting resistance. Therefore, reliable and accurate approaches for the identification of MRDD resistance need to be established. Zhang et al. (2007) reported that maize MRDD plants rated from 0 to 4 at flowering stage, as follows: 0 : healthy plant, $1: 4 / 5-2 / 3$ of healthy plant height, $2: 2 / 3-1 / 2$ of healthy plant height, $3: 1 / 2-1 / 3$ of healthy plant height, $4: 1 / 3$ of healthy plant height or shorter. Indirect enzyme-linked immunosorbent assay (IDELISA) was applied to detect RBSDV in the latest leaf of maize plant: $\mathrm{OD}_{405}$ value of resistant maize plants scoring $0-3$ were 0.391 , $0.487,0.746$, and 0.887 , respectively; $\mathrm{OD}_{405}$ value of moderately resistant maize plants scoring $0-3$ were $0.379,0.517,0.725$, and 0.892 , respectively; $\mathrm{OD}_{405}$ value of susceptible maize plants scoring $0-3$ were $0.387,0.583,0.827,1.026$, and 1.555 , respectively. These studies have suggested that the RBSDV titer determined by ID-ELISA in maize leaves is positively correlated with symptoms in whole plants; the more severe the disease, the higher the virus titer (Zhang et al. 2007). The RBSDV titer could be detected more sensitively and accurately by real-time RT-PCR than by ELISA (Wang et al. 2006).

The goal of this study was to develop an effective method to evaluate resistance to MRDD. In particular, we detected and monitored changes in RBSDV titers in maize seedlings and used a regression analysis of viral replication data to develop an RBSDV replication curve model that will contribute to the precise identification of MRDD resistance and its molecular mechanism.

\section{Materials and Methods}

Plant materials. Eight maize varieties that varied with respect to resistance to MRDD were used, including six inbred lines (Zheng58, Chang7-2, Mo17, Dan340, P138, and Ye478) and one hybrid, Zhengdan958 (Zheng58 $\times$ Chang7-2). The inbred line XH6, whose resistance to MRDD is unknown, was also included in the study.

Laodelphax striatellus, virus transmission, and leaf sample collection. Planthoppers were captured in fields in Lianyungang 
(Jiangsu Province) and fed on susceptible wheat cv. Zhoumai 19 in a pottery flower pot covered with an 80-mesh mothproof net in a greenhouse. The greenhouse was programmed to provide a photoperiod of $14: 10$ (L:D) h with a temperature of $26: 20^{\circ} \mathrm{C}$. The planthoppers completed more than 10 life cycles before they were used in the transmission experiments.

Maize is not a suitable host for planthoppers; when planthoppers are fed on maize plants, they only survive for 5 to 7 days. Accordingly, the second instar virus-free planthoppers were fed on maize MRDD plants obtained in Zhengzhou, China for 3 days to acquire the virus, and were then kept on young wheat seedlings for 7 to 10 days, during which time the planthoppers developed into imagoes with wings. Thirty planthoppers were randomly selected and used for RNA extraction before inoculation on maize seedlings. Uninfected two-leaf maize seedlings grown in pottery flower pots with vermiculite were inoculated for 3 days with 10 viruliferous planthoppers per plant.

There were three flower pots, each with 10 plants for each maize variety, and every experiment was repeated three times. The conditions were $14 \mathrm{~h}$ of daylight with a temperature of $28: 20^{\circ} \mathrm{C}$. Following inoculation, the infected maize plants were sprayed with $5 \%$ imidacloprid (1-(6-chlorine-3 pyridine methyl)- $N$-nitroalkane-2-amino).

The 10 uppermost fully expanded leaves of maize in each flower pot were collected on 7 days, 14 days, 21 days, 28 days, and 35 days after the removal of viruliferous planthoppers.

RNA extraction and cDNA preparation. Total RNAs from the leaves and a single planthopper were extracted using the ZYMO RESEARCH Direct-zol RNA MiniPrep Kit (Irvine, CA, USA) according to the manufacturer's protocol. Reverse transcription kit was purchased from TAKARA (D6110A; Kusatsu, Japan). RNA $(1 \mu \mathrm{g}), 1 \mu \mathrm{l}$ of $10 \mu \mathrm{M}$ RBSDV-R primer, and $1 \mu \mathrm{l}$ of dNTP were added to RNase-Free Water to a total volume of $10 \mu l$. The mixture was incubated at $65^{\circ} \mathrm{C}$ for $5 \mathrm{~min}$ and cooled on ice for $10 \mathrm{~min}$, after which $4 \mu \mathrm{l}$ of $5 \times$ PrimeScripe Buffer, $0.5 \mu l$ of Primescripe RTase, $0.5 \mu l$ of RNase inhibitor, and $5 \mu l$ of RNase-Free Water were added for first-strand cDNA synthesis. The mixture was incubated at $42^{\circ} \mathrm{C}$ for $1 \mathrm{~h}, 95^{\circ} \mathrm{C}$ for $5 \mathrm{~min}$, then $4^{\circ} \mathrm{C}$ for $10 \mathrm{~min}$.

Standard sample and standard calibration curve preparation. The S7 fragment of RBSDV was amplified using the primers RBSDV-F, 5'-AGAGCTCTTCTAGTTATTGCG-3', and RBSDVR, 5'-TCAGCAAAAGGTAAAGGAACG-3', recovered, and ligated to the pMD18-T vector. The primers RBSDV-F and RBSDV-R, named P2 and P1, respectively, were designed by Wu et al. (2000). The two primers were predicted to amplify a 510-bp fragment. After sequencing, the confirmed recombinant plasmid DNA fragment was measured using the NanoDrop 2000 to calculate the plasmid titer, and the initial copy number of the standard sample was $2.98 \times 10^{8}$ copies $/ \mu 1$. A 10-fold of a serial dilution of the standard sample consisted of six solutions with the titers of $2.98 \times 10^{7}$ copies $/ \mu 1,2.98 \times$ $10^{6} \mathrm{copies} / \mu \mathrm{l}, 2.98 \times 10^{5} \mathrm{copies} / \mu \mathrm{l}, 2.98 \times 10^{4}$ copies $/ \mu 1,2.98 \times 10^{3}$ copies $/ \mu 1$, and $2.98 \times 10^{2}$ copies $/ \mu 1$. Fluorescence quantitative RTPCR was performed using the RBSDV-R and RP (5'-GCTCCTACT GAGTTGCCTGTC- $3^{\prime}$ ) primers. The primer RP based on the 510-bp segment was designed by Wang et al. (2006). By the RBSDV-R + RP primer combination, a predicted PCR product of $342 \mathrm{bp}$ was amplified. The standard calibration curve was obtained by plotting the logarithm of the initial copy number of serial dilutions of the recombinant plasmid on the horizontal axis against threshold cycles $(C t)$ on the vertical axis. The viral titer of a plant was quantified by comparing the $C t$ value with the standard curve.

Fluorescence quantitative RT-PCR. The $20-\mu l$ real-time PCR reaction system included: $10 \mu \mathrm{l}$ of $2 \times \mathrm{qPCR}$ master mix (Promega, Madison, WI, USA), $1.0 \mu \mathrm{l}$ of cDNA template, $0.8 \mu \mathrm{l}$ of $10 \mu \mathrm{M}$ gene-specific primer, and $8.2 \mu \mathrm{l}$ of $\mathrm{ddH}_{2} \mathrm{O}$.

Amplification conditions were as follows: initial denaturation at $95^{\circ} \mathrm{C}$ for $2 \mathrm{~min}$, followed by 40 cycles of denaturation at $95^{\circ} \mathrm{C}$ for $15 \mathrm{~s}$, annealing at $60^{\circ} \mathrm{C}$ for $15 \mathrm{~s}$, and extension at $72^{\circ} \mathrm{C}$ for $20 \mathrm{~s}$. Fluorescence quantitative RT-PCRs were performed using the Eppendorf Mastercycler ep realplex. The specific primer was RP and RBSDV-R.

Model Fitting Method Associations between the number of copies of RBSDV and sampling time were analyzed by logistic and linear regression (SPSS, version 19.0). The $\mathrm{F}$

$$
\left(F=\frac{U / f_{U}}{Q_{e} / f_{e}}\right)
$$

and $P$ values were used to demonstrate the goodness of fit.

\section{Results}

Analysis of the rate of viruliferous SBPH. Viruliferous planthoppers were obtained by feeding on RBSDV-infected maize plants

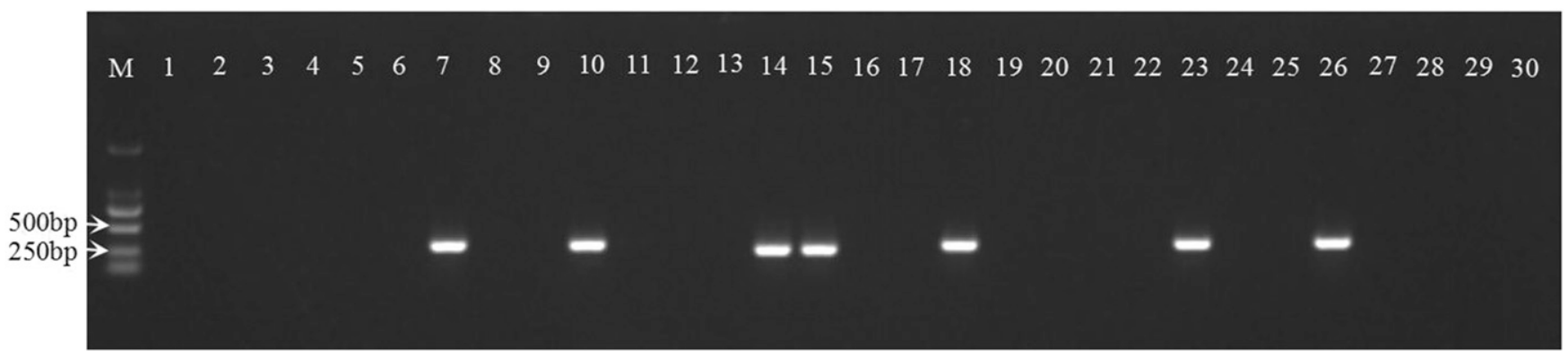

Fig. 1. The rate of small brown planthopper (SBPH) infection by Rice black-streaked dwarf virus (RBSDV) detected by PCR.

Table 1. Viral titer of eight maize varieties in different times (no. days after removal of viruliferous planthoppers)

\begin{tabular}{lcccccr}
\hline Plant materials & $\mathbf{7}(\mathbf{c o p y} / \boldsymbol{\mu l})$ & $\mathbf{1 4}(\mathbf{c o p y} / \boldsymbol{\mu l})$ & $\mathbf{2 1}(\mathbf{c o p y} / \boldsymbol{\mu l})$ & $\mathbf{2 8}(\mathbf{c o p y} / \boldsymbol{\mu l})$ & $\mathbf{3 5}(\mathbf{c o p y} / \boldsymbol{\mu l})$ & Multiplication of the RBSDV $^{\mathbf{b}}$ virus \\
\hline Ye478 & $2.05 \pm 0.11 \times 10^{4}$ & $9.41 \pm 4.48 \times 10^{4}$ & $3.72 \pm 0.42 \times 10^{5}$ & $2.03 \pm 0.83 \times 10^{6}$ & $6.09 \pm 2.37 \times 10^{6}$ & 297.67 \\
XH6 & $1.90 \pm 0.69 \times 10^{4}$ & $6.80 \pm 0.61 \times 10^{4}$ & $2.57 \pm 1.32 \times 10^{5}$ & $5.58 \pm 0.99 \times 10^{5}$ & $4.77 \pm 1.35 \times 10^{6}$ & 250.51 \\
Zhengdan958 & $1.77 \pm 0.25 \times 10^{4}$ & $5.88 \pm 3.02 \times 10^{4}$ & $1.49 \pm 0.86 \times 10^{5}$ & $7.31 \pm 2.51 \times 10^{5}$ & $3.16 \pm 1.09 \times 10^{6}$ & 178.02 \\
Mo17 & $2.69 \pm 0.17 \times 10^{4}$ & $8.41 \pm 4.57 \times 10^{4}$ & $2.06 \pm 0.53 \times 10^{5}$ & $7.99 \pm 1.42 \times 10^{5}$ & $4.17 \pm 0.75 \times 10^{6}$ & 154.88 \\
Zheng58 & $2.64 \pm 0.42 \times 10^{4}$ & $6.76 \pm 3.13 \times 10^{4}$ & $1.94 \pm 1.27 \times 10^{5}$ & $5.58 \pm 4.59 \times 10^{5}$ & $3.59 \pm 1.21 \times 10^{6}$ & 136.28 \\
Dan340 & $4.66 \pm 2.12 \times 10^{2}$ & $5.50 \pm 1.47 \times 10^{2}$ & $1.05 \pm 0.40 \times 10^{3}$ & $2.24 \pm 1.14 \times 10^{3}$ & $2.60 \pm 0.61 \times 10^{3}$ & 5.59 \\
Chang7-2 & $5.22 \pm 1.04 \times 10^{2}$ & $6.36 \pm 3.17 \times 10^{2}$ & $8.55 \pm 2.57 \times 10^{2}$ & $1.82 \pm 0.88 \times 10^{3}$ & $1.83 \pm 0.49 \times 10^{3}$ & 3.52 \\
P138 & $3.86 \pm 1.28 \times 10^{2}$ & $4.57 \pm 3.23 \times 10^{2}$ & $5.13 \pm 0.79 \times 10^{2}$ & $5.89 \pm 1.77 \times 10^{2}$ & $6.55 \pm 1.23 \times 10^{2}$ & 1.70 \\
\hline
\end{tabular}

${ }^{a}$ Values represent the means \pm SD from three replicates.

${ }^{\mathrm{b}}$ Rice black-streaked dwarf virus. 
for $72 \mathrm{~h}$ followed by rearing for another 7 to 10 days to allow the virus to pass through the circulation before inoculation on maize seedlings. Thirty planthoppers were randomly selected. The RNA of each planthopper was extracted and amplified using the RP and RBSDV$\mathrm{R}$ primers for further test in $1 \%$ agarose gels. The rate of viruliferous SBPH was 23.3\% (7/30) (Fig. 1).

Viral titer in resistant maize increased more slowly. RBSDV was detected in leaves of all eight maize varieties after inoculation with viruliferous planthoppers, but there was variation in the viral titers and replication rates. Compared with those of Dan340, P138, and Chang7-2, the viral titers of Ye478, XH6, Mo17, Zheng58, and Zhengdan958 were much greater and increased more dramatically over time. The plants of Ye478, XH6, Mo17, Zheng58, and Zhengdan 958 at 35 days after removal of the viruliferous planthoppers were $297.67,250.51,178.02,154.88$, and 136.28 times the viral titer of the first sampling, respectively. By contrast, at 35 days, the viral titers for Dan340, Chang7-2, and P138 were as much as 5.59, 3.52 , and 1.70 times, respectively, compared with the first sampling

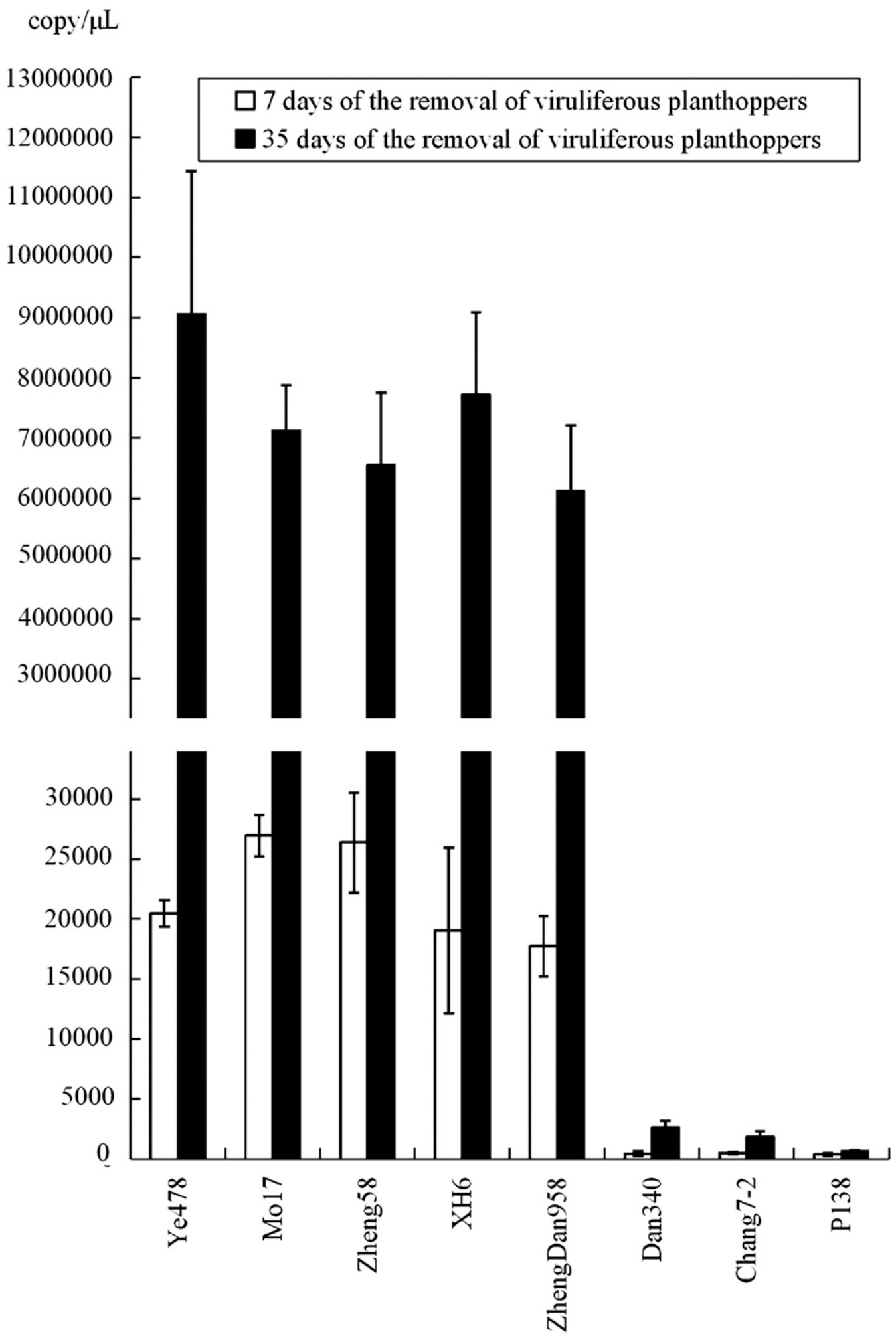

Fig. 2. Comparison of viral titer for first and last sampling. Black bars represent the viral titers (the first sampling) of eight maize varieties at 7 days after the removal of viruliferous planthoppers. White bars represent the viral titers (the last sampling) of eight maize varieties at 35 days after the removal of viruliferous planthoppers. Values represent the means \pm SD from three replicates. 
(Table 1, Fig. 2). These results suggested that viral replication in Dan340, Chang7-2, and P138 was inhibited (Fig. 3).

Standard calibration curve. A standard calibration curve was obtained using standard samples, as shown in Figure 4. The linear regression equation for the standard calibration curve was $Y=-3.17 X+$ $39.38\left(R^{2}=0.997\right)(X$ represents the logarithm of the copy number of the plasmid in each serial dilution and $Y$ is the threshold cycle value). The coefficient of determination $\left(R^{2}=0.997\right)$ indicated a high accuracy for the linear relationship. The threshold cycle $(C t)$ values obtained from the standard calibration curve ranged from 16 to 32 , within the required range of $C t \leq 40$. The detection sensitivity was 298 copies/ $\mu$ l, suggesting that the method was highly sensitive.

Regression analysis of the RBSDV replication curve. Associations between the number of copies of RBSDV and sampling time were analyzed by logistic and linear regression (SPSS, version 19.0). A logistic model fitted the RBSDV replication data for five susceptible varieties, Ye478, Mo17, Zheng58, Zhengdan958, and XH6. The $F$ values for Ye478, Mo17, Zheng58, and Zhengdan958

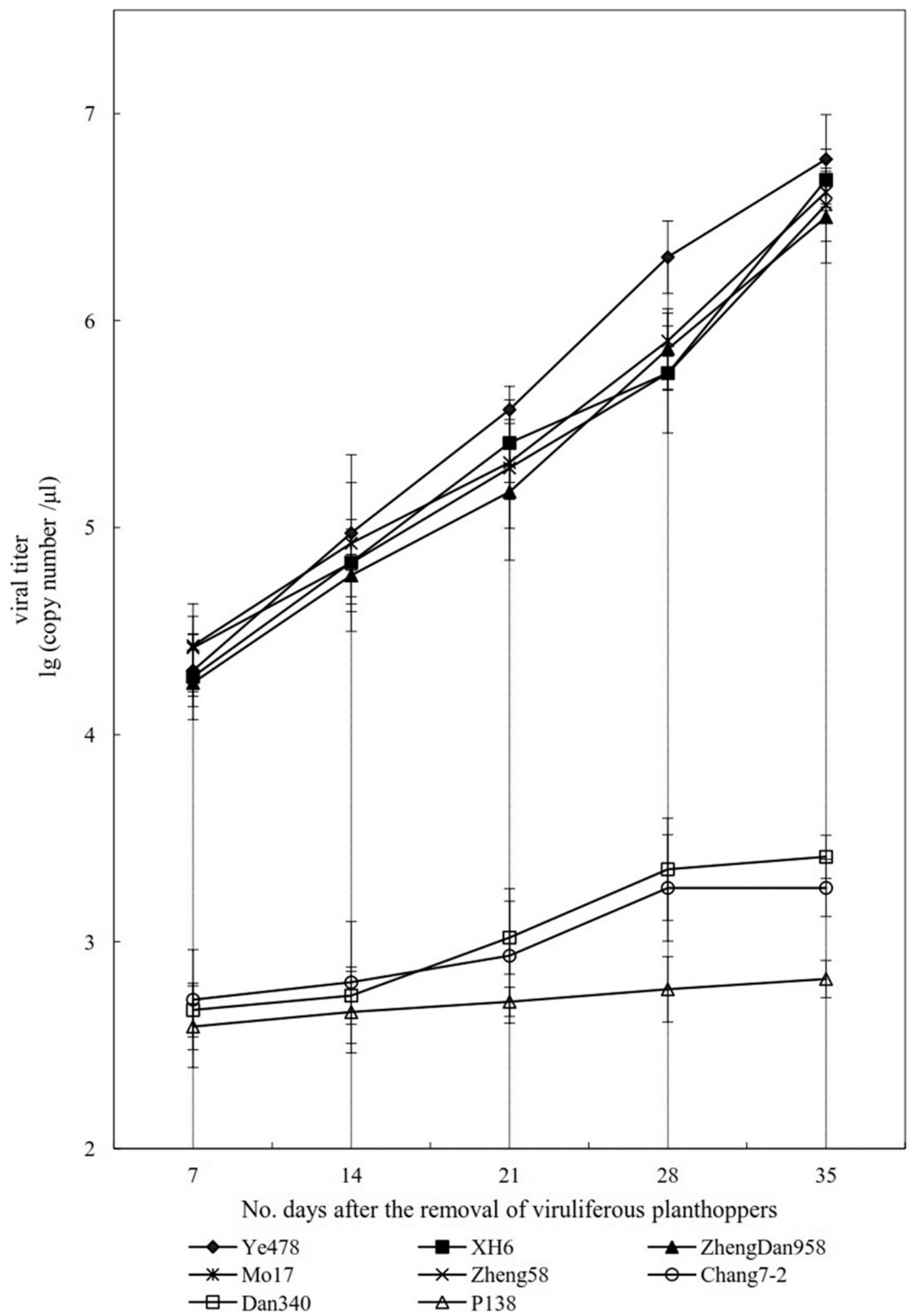

Fig. 3. Comparison of viral titer of eight maize varieties in different times. Horizontal axis shows days when samplings occurred. Vertical axis shows the copy number of virus. Leaf samples were collected at 7 days, 14 days, 21 days, 28 days, and 35 days after the removal of viruliferous planthoppers. 
exceeded $F_{0.01}(2,2)=99.00$, indicating that the logistic model fit the data well. The $F$ value for XH6 was greater than $F_{0.05}(2,2)=19.00$, suggesting that the logistic model fit the data (Table 2).

In the logistic model, a smaller $t_{I P}$, defined as the time point at which the maximum replication rate was detected, corresponded to faster viral reproduction and thus lower resistance to MRDD. Ye478 and XH6, with the lowest $t_{I P}$ values, 33.01 and 33.12, were both categorized as highly susceptible. Mo17, Zheng58, and Zhengdan 958 were classified as susceptible, with $t_{I P}$ values of 48.46, 46.73, and 43.99, respectively (Table 2).

A linear model fit the data for the three resistant varieties; the model fit the data for P138 and D340 $\left(F>F_{0.01}(1,3)=34.12\right)$ and Chang7-2 well $\left(F>F_{0.05}(1,3)=10.13\right)$ (Table 3$)$. The lower the slope of the fitted line, $b$, the slower the replication of the virus and the stronger the resistance. P138, with the lowest $b$ value, 9.56, was highly resistant to MRDD, followed by Chang7-2 and Dan340.

\section{Discussion}

Shen (2014) suggested that RBSDV is distributed throughout the maize plant, with the highest virus titer in roots, followed by leaves, and the lowest in stems. Wu et al. (2000) reported that RBSDV is detected in roots and leaves of maize. Therefore, leaves are widely used to detect RBSDV in maize. The RBSDV titer determined by fluorescence quantitative RT-PCR and ELISA in maize leaves is positively correlated with symptoms in whole plants; the more severe the disease (shortened plant height and internodes; tassel and ear badly

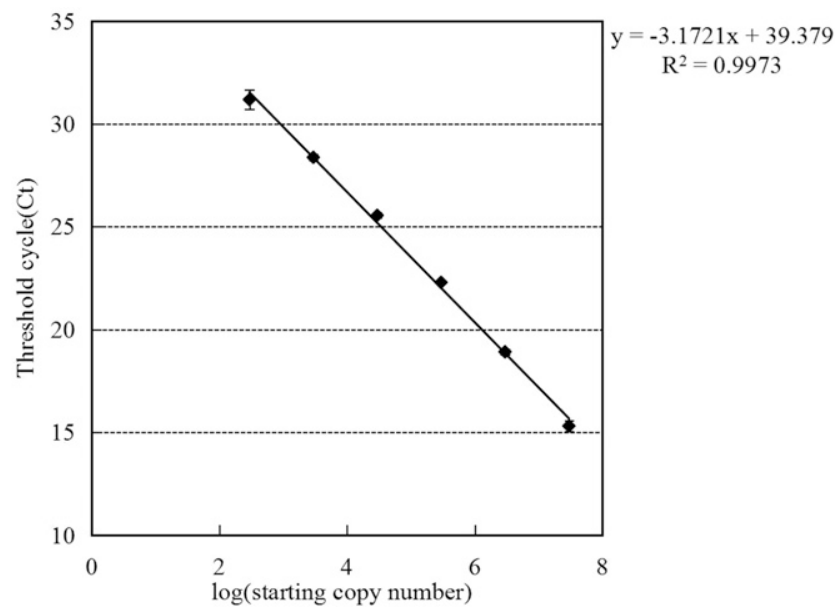

Fig. 4. Standard regression line obtained by plotting $C t$ values versus the logarithm of the starting copy number of plasmid. The $\mathrm{Ct}$ value of each dilution was the mean $\pm \mathrm{SE}$ of three reactions. developed, few or no seeds), the higher the virus titer. There were significant differences on RBSDV titers among the different disease severity graded plants of the same variety (Wang et al. 2006; Zhang et al. 2007). Although the RBSDV titer in leaves is positively correlated with symptoms in whole plants, it cannot be used to evaluate the resistance of the plant itself. We developed an RBSDV replication model to find new reliable molecular markers to evaluate MRDD resistance.

In this study, based on a regression analysis, RBSDV replication in maize seedlings could be described by logistic and linear replication models for maize varieties with different levels of resistance to MRDD. The logistic model fit RBSDV replication data for four varieties previously identified as susceptible, i.e., Ye 478, Mo17, Zheng58, and Zhengdan 958, as well as XH6, whose resistance to MRDD is unknown. There was significant variation in $t_{I P}$ among these five varieties: Ye478 and XH6 had relatively low $t_{I P}$ values, indicating rapid virus replication. Therefore, these varieties were highly susceptible to MRDD. Mo17, Zheng58, and Zheng958 had significantly higher $t_{I P}$ values than those of other varieties, suggesting that the viral reproduction was relatively low and thus these varieties had low susceptibility to MRDD. Therefore, $t_{I P}$ can precisely distinguish between highly susceptible and susceptible varieties. This model can also be applied to varieties with unknown resistance to MRDD, such as XH6.

The linear model fit the data for three resistant varieties, P138, Dan340, and Chang7-2, but with substantially different slopes. The slope of the fitted line can be used to precisely and accurately characterize maize germplasm with different levels of resistance. The highly resistant variety P138 had a significantly lower slope than those of the other two varieties. Although both were previously identified as resistant to MRDD, Chang7-2, with a slope lower than that of Dan340 (54.45 vs. 85.23), showed superior resistance to MRDD. Dan340 was identified as highly susceptible to RBSDV by Lu et al. (2001), but was reported as resistant by Chen et al.(2006). The results of this study showed that a linear model fit the virus replication data for Dan340, suggesting that the maize variety harbors genes that suppress the replication of RBSDV. As a result, Dan340 can be used as resistant germplasm for breeding programs and for further gene mapping and cloning. Thus, the regression analysis of the RBSDV replication curve can help to minimize the loss of germplasm of moderate resistance and will facilitate the utilization of resistant genes and studies of mechanisms underlying resistance to MRDD.

In this study, Chang7-2 was identified as moderately resistant to MRDD and Zheng58 was identified as susceptible, but their hybrid, Zhengdan958, was susceptible. One possible explanation is that the susceptible gene in Zheng58 and the resistance gene in Chang7-2 have epistatic effects. To improve the resistance of the hybrid, its parents should be resistant to MRDD, or at least one parent should be highly resistant.

Table 2. Logistic fitting parameters of Rice black-streaked dwarf virus (RBSDV) replication curve in susceptible materials

\begin{tabular}{|c|c|c|c|c|c|}
\hline & Ye478 $^{a}$ & Mo17 $^{b}$ & XH6 & Zhengdan958 $^{\mathrm{d}}$ & Zheng58 \\
\hline$\overline{t_{I P} \mathrm{f}}$ & 33.01 & 48.46 & 33.12 & 43.99 & 46.73 \\
\hline$F$ & 20546.51 & 2018.55 & 22.79 & 8561.92 & 643.42 \\
\hline$r$ & 0.99998 & 0.99975 & 0.97875 & 0.99994 & 0.99922 \\
\hline$R^{2}$ & 0.99995 & 0.99950 & 0.95796 & 0.99988 & 0.99845 \\
\hline $\mathrm{SS}_{\mathrm{y}}$ & 26581413.29 & 12510735.22 & 16715422.22 & 7149063.65 & 9330894.50 \\
\hline $\mathrm{Q}_{\mathrm{e}}$ & 1293.66 & 6194.81 & 702755.70 & 834.89 & 14479.46 \\
\hline$f_{\mathrm{R}}$ & 2 & & & & \\
\hline$f_{\mathrm{e}}$ & 2 & & & & \\
\hline
\end{tabular}

${ }^{\mathrm{a}}$ RBSDV growth curve model of Ye478: $\mathrm{C}=\frac{\mathrm{A}}{1+\mathrm{Be}} \mathrm{e}^{-\mathrm{kl}}=\frac{9693.0140}{1+6343.6475 \mathrm{e}^{-0.26521}}$.

${ }^{\mathrm{b}}$ RBSDV growth wcurve model of Mo17: $\mathrm{C}=\frac{\mathrm{A}}{1+\mathrm{Be}^{-\mathrm{kt}}}=\frac{104365.1553}{1+93501.8149 \mathrm{e}^{-0.23621}}$.

${ }^{c}$ RBSDV growth curve model of XH6: $\mathrm{C}=\frac{\mathrm{A}}{1+\mathrm{Be}^{-\mathrm{kt}}}=\frac{6932.9803}{1+13460.8181^{-0.2871}}$.

${ }^{\mathrm{d}}$ RBSDV growth curve model of Zhengdan958: $\mathrm{C}=\frac{\mathrm{A}}{1+\mathrm{Be}-\mathrm{kl}}=\frac{26578.2084}{1+18185.0570 \mathrm{e}^{-0.22301}}$.

${ }^{\mathrm{e}} \mathrm{RBSDV}$ growth curve model of Zheng58: $\mathrm{C}=\frac{\mathrm{A}}{1+\mathrm{Be}-\mathrm{kt}}=\frac{81415.3038}{1+209475.0293^{-0.26221}}$.

f $r_{0.05}(2,2)=0.975 ; F_{0.01}(2,2)=99.00 ; F_{0.05}(2,2)=19.00$. C, viral titer; A, growth limit; $\mathrm{B}$, initial growth parameter; $\mathrm{K}$, growth rate parameter; $t_{I P}$, a time point with the maximum growth rate; $t_{I P}=\ln (\mathrm{B}) / \mathrm{K} ; r$, correlation coefficient; $R^{2}$, coefficient of determination; $\mathrm{SS}_{\mathrm{y}}$, total variation; $\mathrm{Q}_{\mathrm{e}}$, sum of the squared errors; $f_{\mathrm{R}}$, regression degrees of freedom; $f_{\mathrm{e}}$, degrees of freedom for error. 
Table 3. Linear fitting parameters of Rice black-streaked dwarf virus (RBSDV) replication curve in resistant materials

\begin{tabular}{lccc}
\hline & Chang7-2 $^{\mathbf{a}}$ & Dan340 $^{\mathbf{b}}$ & $\mathbf{P 1 3 8}^{\mathbf{c}}$ \\
\hline$b^{\mathrm{d}}$ & 54.45 & 85.23 & 9.56 \\
$F$ & 20.58 & 34.45 & 1950.34 \\
$r$ & 0.934 & 0.959 & 0.999 \\
$R^{2}$ & 0.873 & 0.920 & 0.998 \\
$\mathrm{Q}_{\mathrm{e}}$ & 211717.63 & 309983.01 & 68.88 \\
$\mathrm{SS}_{\mathrm{y}}$ & 1664402.05 & 3869495.48 & 44849.22 \\
$f_{\mathrm{R}}$ & 1 & & \\
$f_{\mathrm{e}}$ & 3 & & \\
\hline
\end{tabular}

${ }^{a}$ RBSDV growth curve model of Chang7-2: $C=b x+a=54.4487 x-9.3849$.

${ }^{b}$ RBSDV growth curve model of Dan340: $C=b x+a=85.2309 x-406.6198$.

${ }^{c}$ RBSDV growth curve model of P138: $\mathrm{C}=\mathrm{bx}+\mathrm{a}=9.5597 \mathrm{x}+319.2888$.

${ }^{\mathrm{d}} r_{0.05}(1,3)=0.8783 ; F_{0.05}(1,5-2)=10.13 ; F_{0.1}(1,5-2)=5.54$. C, viral titer; $b$, regression coefficients; $r$, correlation coefficient; $R^{2}$, coefficient of determination; $\mathrm{Q}_{\mathrm{e}}$, residual sum of squares; $\mathrm{SS}_{\mathrm{y}}$, total variation; $f_{\mathrm{R}}$, regression degrees of freedom; $f_{\mathrm{e}}$, degrees of freedom for error.

The variation in maize resistance to MRDD is manifested by differences in the cumulative replication of RBSDV in plants. The replication curve of RBSDV in seedlings after inoculation can be used to distinguish germplasm from highly susceptible, susceptible, moderately resistant, and resistant varieties. Therefore, this new method can be used to evaluate MRDD resistance and can promote the use of MRDD-resistant germplasm and the cultivation of resistant varieties.

\section{Acknowledgments}

XHH and SGT designed the study. RQY, SLG, and MMX performed the experiments. TMC and XHH analyzed the data and drafted the manuscript. XLF, HFC, and XHG assisted with the data analysis and aided in writing the manuscript. CXL, YPF, NNC, and LL aided in performing the experiments. XHH and SGT modified the manuscript. All of the authors carefully checked and approved this version of the manuscript.

\section{Literature Cited}

Chen, C. J., Cheng, Z. B., and Xiong, R. Y. 2004. Model of rice black streaked dwarf virus (RBSDV) transmission on maize seedling by Laodelphax striatellus Fallen. J. Shanghai Jiaotong Univ. Agric. Sci. 22:7-11 (in Chinese with English abstract).

Chen, Y. K., Li, X. H., and Xiao, M. J. 2006. Genetic variation in sixty-four maize inbred lines in relation to maize rough dwarf virus. Acta Agron. Sin. 32: 1848-1854 (in Chinese with English abstract).
Chen, Y. P., Yuan, J. H., Meng, Q. C., and Zhang, Y. B. 2007. Study on identification for the resistance of maize inbred lines to maize rough dwarf virus. J. Jinling Inst. Technol. 23:57-60 (in Chinese with English abstract).

Di Renzo, M. A., Bonamico, N. C., Diaz, D. G., Ibanez, M. A., Faricelli, M. E., Balzarini, M. G., and Salerno, J. C. 2004. Microsatellite markers linked to QTL for resistance to Mal de Rio Cuarto disease in Zea mays L. J. Agric. Sci. 142:289-295.

Fang, S., Yu, J., Feng, J., Han, C., Li, D., and Liu, Y. 2001. Identification of rice black-streaked dwarf fijivirus in maize with rough dwarf disease in China. Arch. Virol. 146:167-170.

Lenardon, S. L., March, G. J., Nome, S. F., and Ornaghi, J. A. 1998. Recent outbreak of "Mal de Rio Cuarto" virus on corn in Argentina. Plant Dis. 82:448.

Louie, R. 1999. Diseases caused by viruses. Pages 49-55 in: Compendium of Corn Diseases. D. G. White, ed. American Phytopathological Society, St. Paul, MN.

Lu, Y. G., Di, D. P., Miao, H. Q., and Tian, L. Z. 2001. Identification and analysis on resistance of introduced foreign and domestic maize inbreeds to MRDV. J. Hebei Agric. Sci. 5:21-25 (in Chinese with English abstract).

Luan, J. W., Wang, F., Li, Y. J., Zhang, B., and Zhang, J. R. 2012. Mapping quantitative trait loci conferring resistance to rice black-streaked virus in maize (Zea mays L.). Theor. Appl. Genet. 125:781-791.

Shen, J. F. 2014. Characterization of morphology and gene expression changes in tumor-like structure mediated by rice black-streaked dwarf virus infection. [D]. Zhejiang Normal University, Jinhua.

Shi, L. Y., Hao, Z. F., Weng, J. F., Xie, C. X., Liu, C. L., Zhang, D. G., Li, M. S., Bai, L., Li, X. H., and Zhang, S. H. 2012. Identification of a major quantitative trait locus for resistance to maize rough dwarf virus in a Chinese maize inbred line X178 using a linkage map based on 514 gene-derived single nucleotide polymorphisms. Mol. Breed. 30:615-625.

Wang, F., Qin, G. Z., Sui, Z. H., Wang, Z. H., Wang, Z. Y., Yu, J. L., and Zhang, J. R. 2006. Improved method for assaying maize plant resistance to maize rough dwarf disease by artificial inoculation and real-time RT-PCR. Eur. J. Plant Pathol. 116:289-300.

Wu, S. H., Wang, Z. H., Fang, Y. J., Zhou, Y. J., Cheng, Z. B., and Zhang, W. C. 2000. Detection of pathogen of maize rough dwarf disease (MRDD) in Jiangsu province with RT-PCR. J. Agric. Biotechnol. 8:369-372 (in Chinese with English abstract).

Xie, S. X., He, D. Y., Cen, M. Y., and Feng, L. S. 2009. Resistance identification and analysis of the maize germplasm to MRDV. Bull. Agric. Sci. Technol. 12: 50-52 (in Chinese with English abstract).

Xue, L., Zhang, D., and Xu, L. 2011. Mining and analyzing genetic diversity for maize rough dwarf disease resistant germplasms and its application in maize breeding. Acta Agron. Sin. 37:2123-2129 (in Chinese with English abstract).

Zhang, A. H., Miao, H. Q., Zhu, B. C., Lu, Y. G., Chen, D., and Di, D. P. 2007. Studies on the titer of RBSDV in corn inbred lines with different resistance to the disease. J. Hebei Agric. Univ. 30:79-82 (in Chinese with English abstract).

Zhang, H. M., Chen, J. P., Lei, J. L., and Adams, M. J. 2001. Sequence analysis shows that a dwarfing disease on rice, wheat and maize in China is caused by rice black-streaked dwarf virus. Eur. J. Plant Pathol. 107:563-567.

Zhou, Y. J., Wang, Z. R., Hou, Q. S., Zhang, J. X., Wu, S. H., Cheng, Z. B., and Fan, Y. J. 1996. Outbreak of a maize dwarf disease caused by RBSDV in Jiangsu. Jiangsu Agric. Sci. 12:40-41. 